\title{
Effects of plant sterol esters in skimmed milk and vegetable-fat-enriched milk on serum lipids and non-cholesterol sterols in hypercholesterolaemic subjects: a randomised, placebo-controlled, crossover study
}

\author{
Patricia Casas-Agustench ${ }^{1,2}$, Mercè Serra ${ }^{1,2}$, Ana Pérez-Heras ${ }^{1,2}$, Montserrat Cofán ${ }^{1,2}$, Xavier Pintó 3 , \\ Elke A. Trautwein ${ }^{4}$ and Emilio Ros ${ }^{1,2 *}$ \\ ${ }^{1}$ Lipid Clinic, Service of Endocrinology and Nutrition, Institut d'Investigations Biomediques August Pi Sunyer, \\ Hospital Clinic, Villarroel 170, 08036 Barcelona, Spain \\ ${ }^{2}$ Ciber Fisiopatología de la Obesidad y Nutrición (CIBERobn), Instituto de Salud Carlos III (ISCIII), Spain \\ ${ }^{3}$ Lipids and Vascular Risk Unit, Department of Internal Medicine, Hospital Universitari de Bellvitge, L'Hospitalet de Llobregat, \\ Barcelona, Spain \\ ${ }^{4}$ Unilever Research and Development, Vlaardingen, The Netherlands \\ (Submitted 22 March 2011 - Final revision received 10 August 2011 - Accepted 18 August 2011 - First published online 10 October 2011)
}

\begin{abstract}
Plant sterol (PS)-supplemented foods are recommended to help in lowering serum LDL-cholesterol (LDL-C). Few studies have examined the efficacy of PS-enriched skimmed milk (SM) or semi-SM enriched with vegetable fat (PS-VFM). There is also insufficient information on factors predictive of LDL-C responses to PS. We examined the effects of PS-SM ( $0 \cdot 1 \%$ dairy fat) and PS-VFM (0.1\% dairy fat plus $1 \cdot 5 \%$ vegetable fat) on serum lipids and non-cholesterol sterols in hypercholesterolaemic individuals. In a placebo-controlled, crossover study, forty-three subjects with LDL-C $>1300 \mathrm{mg} / \mathrm{l}$ were randomly assigned to three 4-week treatment periods: control SM, PS-SM and PS-VFM, with $500 \mathrm{ml}$ milk with or without $3.4 \mathrm{~g}$ PS esters (2 g free PS). Serum concentrations of lipids and non-cholesterol sterols were measured. Compared to control, LDL-C decreased by 8.0 and $7.4 \%(P<0.015$, both $)$ in the PS-SM and PS-VFM periods, respectively. Serum lathosterol:cholesterol (C) ratios increased by 11-25\%, while sitosterol:C and campesterol:C ratios increased by $70-120 \%$ with both the PS-fortified milk. Adjusted LDL-C reductions were variably enhanced in participants with basal low serum lathosterol/C or conversely high sitosterol/C and campesterol/C. Subjects with post-treatment serum PS:C ratios above the median showed mean LDL-C changes of -5.9 to $-10.4 \%$, compared with 1.7 to $-2.9 \%$ below the median. In conclusion, consumption of $2 \mathrm{~g} / \mathrm{d}$ of PS as PS-SM and PS-VFM lowered LDL-C in hypercholesterolaemic subjects to a similar extent. Basal and post-treatment changes in markers of cholesterol metabolism indicating low cholesterol synthesis and high cholesterol absorption predicted improved LDL-C responses to PS.
\end{abstract}

Key words: Plant sterols: Dairy products: Cholesterol metabolism: Non-cholesterol sterols: Cholesterol lowering

Plant sterols (PS) are established non-pharmacological agents that help reduce serum total and LDL-cholesterol (LDL-C) concentrations in conjunction with a healthy diet. PS are naturally occurring plant constituents that are structurally similar to cholesterol and partially inhibit its intestinal absorption, which is the mechanism responsible for their cholesterol-lowering effect. Although details are not fully elucidated, it is believed that displacement of cholesterol from intestinal micelles by the more hydrophobic and bulkier PS molecules limits the amount available for absorption, resulting in less cholesterol reaching the liver with ensuing stimulation of LDL clearance ${ }^{(1,2)}$.

According to recent meta-analyses of randomised controlled trials in adults, the predicted LDL-C-lowering effect of the recommended daily dose of PS or plant stanols (approximately $2 \mathrm{~g}$ ) is about 9 to $10 \%{ }^{(3,4)}$. Theoretically, the food or beverage format used as the matrix for PS could influence the magnitude of the cholesterol-lowering response. In their meta-analysis, Demonty et al. ${ }^{(3)}$ systematically evaluated the effect of food format and, at standard doses of PS, found no differences in efficacy between high-fat $v$. low-fat and solid $v$. liquid foods. In another meta-analysis, AbuMweis et $a l .{ }^{(5)}$ suggested that PS or stanols consumed in fat spreads, mayonnaise and salad dressings, and milk and yoghurt would lead to larger pooled average LDL-C reductions than other food formats. Together, with the fact that some dairy foods are low in fat, these data suggest that fat content is

Abbreviations: BP, blood pressure; C, cholesterol; HDL-C, HDL-cholesterol; LDL-C, LDL-cholesterol; PS, plant sterols; SM, skimmed milk; VFM, vegetable fat-enriched milk. 
not a crucial characteristic for ensuring optimal efficacy of PS-enriched foods.

However, it appears to be counterintuitive to use highfat food products as vehicles for a cholesterol-lowering agent in the diet of hypercholesterolaemic subjects. If PS dispersed in low-fat drinks retain their cholesterol-lowering efficacy, then either skimmed dairy products or those with replacement of dairy fat by or enrichment with vegetable fat appear as best suited. Given its enrichment in unsaturated fatty acids, dairy products containing vegetable fat might even enhance the cholesterol-lowering response to PS. So far, only a few studies have examined the cholesterol-lowering effect of PS-fortified milk or yoghurt; fewer were considered goodquality studies in the cited meta-analysis ${ }^{(3)}$; and no study has compared the serum lipid effects of skimmed milk (SM) and semi-skimmed vegetable-fat-enriched milk (VFM) containing PS.

A common observation in clinical practice is that there is a significant and large inter-individual variability in the extent of cholesterol reduction to a PS challenge in spite of using the same food format, daily dose, intake occasion and timing in compliant individuals, whereby responders and non-responders are often identified. Cholesterol absorption efficiency also shows a large inter-individual variability, which ranges from 20 to $80 \%{ }^{(6)}$ and is ascribable in part to variation in genes involved in the metabolic pathways along the enterohepatic axis ${ }^{(7)}$. Circulating non-cholesterol sterols can be used as surrogate markers of cholesterol metabolism in such a way that levels of the main cholesterol precursor, lathosterol, reflect endogenous synthesis, while those of PS such as sitosterol and campesterol mirror the efficiency of intestinal absorption $^{(8)}$. It has been postulated that individuals who disclose high basal serum PS levels (being cholesterol hyper-absorbers) should benefit more from an inhibition of intestinal cholesterol absorption by PS or plant stanols, while the converse would occur in those with low serum PS levels (being cholesterol hypo-absorbers), but the evidence thus far is contradictory, with studies both supporting ${ }^{(9-12)}$ and negating this hypothesis ${ }^{(13,14)}$. On the other hand, it has been recently reported that subjects with high endogenous cholesterol synthesis have a lesser cholesterol-lowering response to PS intake than those with low cholesterol synthesis $^{(15)}$.

We hypothesised that PS-fortified SM with partial enrichment of vegetable fat (PS-VFM with $0 \cdot 1 \%$ dairy fat plus $1.5 \%$ vegetable fat) would lower LDL-C concentrations to a greater extent than skimmed milk (0.1\% dairy fat) with the same PS content (PS-SM) and that basal circulating noncholesterol sterol concentrations and their changes after PS consumption would relate to the variability of LDL-C responses. To test these hypotheses, we conducted a randomised, placebo-controlled, crossover feeding study with hypercholesterolaemic subjects comparing two such PS-enriched milk products with plain SM $(0 \cdot 1 \%$ dairy fat $)$ for outcomes on changes of the serum lipid profile and of serum noncholesterol sterols as surrogate markers of cholesterol synthesis and absorption.

\section{Materials and methods}

\section{Subjects}

Adult men and women with polygenic hypercholesterolaemia attending the Lipid Clinics of the Hospital Clínic of Barcelona and Hospital Universitari de Bellvitge were eligible for participation if they had serum LDL-C concentrations greater than $1300 \mathrm{mg} / \mathrm{l}(3.37 \mathrm{mmol} / \mathrm{l})$ and TAG concentrations less than $3000 \mathrm{mg} / \mathrm{l}(3.36 \mathrm{mmol} / \mathrm{l})$; no evidence of alcohol or drug abuse; absence of diabetes mellitus and liver, kidney, thyroid or other endocrine diseases, as assessed by medical history, a complete physical examination and laboratory tests; no history of lactose intolerance; and not being on a weight-reducing diet. Subjects under stable treatment with statins or fibrates were eligible if fulfilling the aforementioned lipid criteria. Consumption within the preceding 8 weeks of other compounds capable of influencing cholesterol metabolism (bile acid-binding resins, ezetimibe, psyllium, fish oil supplements, soya lecithin, phytoestrogens and other PS-fortified foods) was a reason for exclusion. Because the selected study population had common (polygenic) hypercholesterolaemia, we also excluded persons whose elevated serum cholesterol levels had a strong genetic basis (such as heterozygous familial hypercholesterolaemia or familial combined hyperlipidaemia), as established by standard criteria. On admission to the Lipid Clinics, all participants had been advised to follow a Mediterranean-type lipid-lowering diet.

For a crossover design, statistical power calculation indicated that to detect a mean difference of 80 (SD 140) $\mathrm{mg} / \mathrm{l}$ in serum LDL-C between the PS-enriched milk (PS-SM and PSVFM) and the placebo SM, forty-two patients in total would need to complete the study ( $\alpha$ of 0.05 and a power of $>0 \cdot 9)$. From computerised registers of clinical records, fiftysix hypercholesterolaemic patients (twenty-seven women and twenty-nine men) who initially met the eligibility criteria were selected for screening and were asked to participate in the study. They were offered free milk but no monetary compensation.

\section{Study design}

This double-blind, two-centre, placebo-controlled, crossover, randomised study consisted of a 4-week run-in period with general recommendations of a Mediterranean-type lipid-lowering diet and consumption of $500 \mathrm{ml}$ placebo SM in two portions per $\mathrm{d}$ followed by a 12-week experimental phase with the same background diet. At the end of the run-in period, participants were randomly assigned by a computer-generated random number table using a balanced latin-square design to one of six possible diet sequences, consisting of three intervention periods lasting 4 weeks each: placebo SM (control), PS-SM and PS-VFM. Given that diet-induced lipoprotein changes stabilise in $<4$ weeks $^{(16)}$, we did not incorporate a washout period between the diet periods. Subjects were requested to follow the same dietary recommendations throughout the duration of the study and to consume the two daily $250 \mathrm{ml}$ milk servings with two different main meals 
after thoroughly shaking the package to minimise adsorption of PS to its inner surface.

Routine laboratory measurements were conducted at the screening visit to ensure both normal health status and fulfillment of eligibility of lipid criteria. Twice during the last week of the run-in period and of each one of the three dietary periods (control, PS-SM and PS-VFM), the study participants came to the clinic for a medical visit, an interview with the dietitian, anthropometric and blood pressure (BP) measurements, and blood sampling. Adverse effects were recorded at each visit.

The study protocol was approved by the institutional review boards of each institution and all participants provided written informed consent. This study was conducted according to the guidelines laid down in the Declaration of Helsinki and was registered at www.controlled-trials.com as ISRCTN14285706.

\section{Dietary intervention}

All subjects had face-to-face interviews with the dietitian for nutritional counselling to facilitate understanding of the study protocol and adherence to the background diet and study milk consumption. At the end of each treatment period, dietary compliance was assessed by $7-d$ diet recalls. The nutrient composition of the diets was calculated with Food Processor Plus software, version 8.0 (ESHA Research, Salem, OR, USA), which was adapted to the nutrient databases of specific Mediterranean foods when appropriate. Intake of total PS in the usual diet was estimated from the database of Spanish foods developed by Jiménez-Escrig et al. ${ }^{(17)}$.

The PS-fortified and control milk products were produced under factory-controlled conditions by Unilever (Vlaardingen, the Netherlands) and portion packed in $250 \mathrm{ml}$ coded containers. The ultra high temperature shelf-stable milk products were plain, placebo SM ( $0 \cdot 1 \%$ dairy fat), SM enriched with $6.8 \mathrm{~g} / \mathrm{l}$ PS esters (equivalent to $4 \mathrm{~g} / \mathrm{l}$ of free PS) and SM enriched with vegetable fat to the level of a semi-SM $(0 \cdot 1 \%$ dairy fat plus $1.5 \%$ vegetable fat) and $6.8 \mathrm{~g} / 1$ of PS esters. The nutritional composition of the milk products is shown in Table 1. The origin of the PS was tall oil and they were esterified with fatty acids from sunflower oil. The composition of the PS was $78.7 \%$ sitosterol, $8.9 \%$ sitostanol, $7.7 \%$ campesterol, $1 \cdot 1 \%$ stigmasterol and $1 \%$ campestanol, with other minor sterols/stanols making up the remaining $2 \cdot 6 \%$.

Adherence to study milk intake was assessed by recount of empty packages returned to the clinic after each diet period. In addition, serum PS concentrations, which typically increase with PS ingestion, were used as a biological marker of compliance.

\section{Measurements}

BMI was calculated as weight $(\mathrm{kg})$ divided by the square of height $(\mathrm{m})$. Waist circumference was measured after expiration at the midpoint between the lowest rib and the iliac crest. BP was measured with a random-zero mercury sphygmomanometer. We used the mean of two measurements of systolic
Table 1. Nutrient composition of control and plant sterol (PS)-fortified milk products (per $500 \mathrm{ml}$ daily serving)

\begin{tabular}{lccc}
\hline & $\begin{array}{c}\text { Control } \\
\text { skimmed } \\
\text { milk }\end{array}$ & $\begin{array}{c}\text { PS-fortified } \\
\text { skimmed } \\
\text { milk }\end{array}$ & $\begin{array}{c}\text { PS-fortified } \\
\text { semi-skimmed } \\
\text { vegetable-fat milk }\end{array}$ \\
\hline Energy* $^{*}$ & & & \\
kcal & 164 & 176 & 244 \\
kJ & 686 & 736 & 1021 \\
Protein (g) & 15.8 & 15.8 & 15.8 \\
Carbohydrate (g) & 24.0 & 24.0 & 24.0 \\
Total fat (g) & 0.5 & 1.9 & 9.4 \\
Dairy fat (g) & 0.5 & 0.5 & 0.5 \\
Non-dairy fat from & 0.0 & 1.4 & 1.4 \\
fatty acid esters (g) & & & \\
Vegetable fat (g) & 0.0 & 0.0 & 7.5 \\
SFA & 0.4 & 0.5 & 1.4 \\
MUFA* & 0.2 & 0.5 & 2.4 \\
PUFA* & 0.0 & 0.8 & 5.5 \\
PS esters (g) & 0.0 & $3.4 \dagger$ & $3.4 \dagger$ \\
Na (g) & 0.3 & 0.3 & 0.3 \\
Ca (mg) & 600 & 600 & 600 \\
\hline
\end{tabular}

* Includes energy, total fat and fatty acids from PS esters.

† Equivalent to $2.0 \mathrm{~g}$ of free PS.

and diastolic BP taken while subjects were sitting after a 5 min rest.

Venous blood samples were collected and immediately centrifuged at $2500 \mathrm{rpm}$ for $10 \mathrm{~min}$ at $4^{\circ} \mathrm{C}$. All biochemical determinations were performed at the core laboratory in Hospital Clínic. Freshly separated serum was used to determine total cholesterol, TAG and HDL-cholesterol (HDL-C; after precipitation of apo B-containing lipoproteins) by standard enzymatic methods in an automatic analyser DDPPII Hitachi (Roche, Basle, Switzerland), using specific reagents (Boehringer-Mannheim, Mannheim, Germany). LDL-C was calculated by using the Friedewald equation. In one of the duplicate blood samples, concentrations of variables reflecting glucose control and kidney and liver function, i.e. fasting glucose, total bilirubin, aspartate aminotransferase, alanine transaminase, alkaline phosphatase, $\boldsymbol{\gamma}$-glutamyl transpeptidase and creatinine were determined on the automatic analyser. Haematological variables (total and differential leucocyte count, erythrocyte count, $\mathrm{Hb}$ concentration, haematocrit, mean corpuscular volume, and platelet count and volume) were determined on a Coulter counter. None of these variables was affected by the treatments (data not shown).

Serum non-cholesterol sterols were measured by GC, as described before ${ }^{(18)}$. Epicoprostanol $(2 \mu \mathrm{g})$ was added to serum $(0 \cdot 1 \mathrm{ml})$ as internal standard. After alkaline hydrolysis, extraction and derivatisation to trimethylsilyl ethers, sterols were quantified on a 30-m non-polar capillary column (TRBEsterol, Teknokroma, Barcelona, Spain) equipped with flame ionisation detection in a Perkin Elmer GC AutosystemTM (Perkin Elmer, Norwalk, CT, USA) apparatus. Each run quantified the most representative non-cholesterol sterols, i.e. lathosterol, campesterol and sitosterol. Non-cholesterol sterols are expressed as both absolute values ( $\mu \mathrm{mol} / \mathrm{l})$ and ratios to cholesterol ( $\mathrm{C}, \mu \mathrm{mol} / \mathrm{mmol}$ ) because, like cholesterol, these sterols are transported exclusively in lipoprotein particles and their concentrations are altered by changes in carrier 
lipoprotein concentrations ${ }^{(1)}$. Inter- and intra-assay $\mathrm{CV}$ were 5.0 and $3.2 \%$ for lathosterol, 1.9 and $1.6 \%$ for campesterol and 2.0 and $1.8 \%$ for sitosterol, respectively.

\section{Statistical analyses}

The two measurements obtained at the end of each dietary period were averaged. Macronutrient, anthropometric and $\mathrm{BP}$ changes among the four treatment periods (run-in, placebo milk and the two PS-fortified milk) were compared by a repeated-measures ANOVA. Bonferroni's correction was used to correct for multiple testing. Treatment order and centre were entered in the models as an additional factor, but they were not significant and were not considered further. Serum lipid and sterol concentrations at the end of the run-in and each intervention period were considered as the baseline value for the following intervention period and data were analysed by using a mixed-model ANCOVA for repeated measures, with sex, age, baseline levels, period, and treatment sequence and their interactions with treatment as covariates. All analyses started with the same model including all main effects and first-order interactions, and then non-significant terms were removed for the final model. The Tukey-Kramer post hoc test was used for multiple comparisons in these analyses. Models considering LDL-C concentrations by groups with high $v$. low non-cholesterol sterols were examined by an ANCOVA with baseline LDL-C and hypolipidaemic drug treatment as covariates. Pearson's correlation coefficients were used to assess relationships between continuous variables. Partial correlations adjusted for LDL-C levels after placebo were used to assess relationships between LDL-C changes and non-cholesterol sterol ratios adjusting by basal LDL-C levels and hypolipidaemic drug treatment. Statistical significance was set at the $P<0.05$ level. Data were analysed using software SPSS 16.0 (SPSS, Inc., Chicago, IL, USA) and SAS version 9.2 (TS1M0; SAS Institute, Inc., Cary, NC, USA).

\section{Results}

\section{Study participants}

Of the fifty-six selected candidates, forty-four accepted to participate in the study and signed the informed consent (twentytwo in each centre). One woman withdrew soon after start of the study for personal reasons. Therefore, forty-three subjects (twenty-two men and twenty-one women) with a mean age of 49 (SD 13) years completed the study and were included in the final analysis. Eight subjects were under statin treatment and one subject was treated with fibrates. Other treatments were

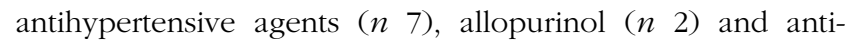
inflammatory agents ( $n$ 2). None of these subjects discontinued drug treatment or changed doses during the trial.

\section{Adherence to the dietary intervention}

The self-reported nutrient intakes of both the run-in and experimental diet periods showed good adherence to the healthy, Mediterranean-type dietary recommendations. As shown in Table 2, the nutrient composition was similar in all diet periods, except for the PS-VFM period, in which total fat and PUFA content increased at the expense of carbohydrates, reflecting the differential composition of the corresponding VFM. Other nutrients, e.g. protein and total energy intake showed differences that were small but statistically significant. Importantly, the background diet intakes of most nutrients capable of influencing lipid metabolism, i.e. SFA,

Table 2. Dietary composition during each treatment period*

(Mean values and standard deviations)

\begin{tabular}{|c|c|c|c|c|c|c|c|c|c|}
\hline & \multicolumn{2}{|c|}{ Run-in } & \multicolumn{2}{|c|}{$\begin{array}{c}\text { Placebo skimmed } \\
\text { milk }\end{array}$} & \multicolumn{2}{|c|}{$\begin{array}{l}\text { PS-fortified skimmed } \\
\text { milk }\end{array}$} & \multicolumn{2}{|c|}{$\begin{array}{l}\text { PS-fortified semi- } \\
\text { skimmed vegetable } \\
\text { fat milk }\end{array}$} & \multirow[b]{2}{*}{$P$ ANOVA } \\
\hline & Mean & SD & Mean & SD & Mean & SD & Mean & SD & \\
\hline \multicolumn{10}{|l|}{ Total energy† } \\
\hline $\mathrm{kcal} / \mathrm{d}$ & $2029^{a, b}$ & 517 & $1877^{a}$ & 424 & $2010^{a, b}$ & 436 & $2045^{\mathrm{b}}$ & 487 & 0.032 \\
\hline $\mathrm{kJ} / \mathrm{d}$ & 8489 & 2163 & 7853 & 1774 & 8410 & 1824 & 8556 & 2038 & 0.032 \\
\hline Protein (\% of energy) & $20 \cdot 8^{a, b}$ & 4.2 & $21 \cdot 8^{\mathrm{a}}$ & 3.8 & $19 \cdot 4^{\mathrm{b}}$ & 3.8 & $20 \cdot 4^{b}$ & 3.4 & 0.001 \\
\hline Carbohydrate (\% of energy) & $42 \cdot 8^{\mathrm{a}}$ & 7.5 & $41 \cdot 4^{\mathrm{a}, \mathrm{b}}$ & $7 \cdot 6$ & $42 \cdot 5^{a, b}$ & $8 \cdot 0$ & $39 \cdot 5^{b}$ & $5 \cdot 8$ & 0.038 \\
\hline Total fibre $(g / d)$ & $19 \cdot 3^{a}$ & $9 \cdot 0$ & $16 \cdot 8^{\mathrm{a}}$ & $6 \cdot 4$ & $17 \cdot 3^{\mathrm{a}}$ & $7 \cdot 0$ & $18 \cdot 3^{a}$ & $7 \cdot 2$ & 0.126 \\
\hline Soluble fibre $(g / d)$ & $5 \cdot 0^{a}$ & 2.7 & $4 \cdot 2^{a}$ & 1.9 & $4 \cdot 5^{\mathrm{a}}$ & $2 \cdot 1$ & $4 \cdot 6^{\mathrm{a}}$ & $2 \cdot 3$ & 0.138 \\
\hline Fat (\% of energy) $†$ & $35 \cdot 4^{\mathrm{a}}$ & 6.9 & $35 \cdot 5^{a}$ & $6 \cdot 3$ & $36 \cdot 9^{a, b}$ & $7 \cdot 1$ & $38 \cdot 7^{b}$ & $5 \cdot 7$ & 0.012 \\
\hline SFAt & $7 \cdot 9^{\mathrm{a}}$ & $1 \cdot 7$ & $8 \cdot 3^{a}$ & $2 \cdot 2$ & $8 \cdot 3^{a}$ & $2 \cdot 0$ & $8 \cdot 5^{\mathrm{a}}$ & $2 \cdot 0$ & 0.441 \\
\hline MUFA† & $19 \cdot 2^{\mathrm{a}}$ & $5 \cdot 2$ & $19 \cdot 0^{\mathrm{a}}$ & 4.4 & $20 \cdot 1^{a}$ & $5 \cdot 0$ & $20 \cdot 5^{a}$ & $4 \cdot 2$ & 0.114 \\
\hline PUFAt & $5 \cdot 3^{a}$ & $2 \cdot 2$ & $5 \cdot 5^{\mathrm{a}}$ & 1.7 & $5 \cdot 7^{\mathrm{a}}$ & 1.6 & $7 \cdot 2^{\mathrm{b}}$ & $1 \cdot 1$ & $<0.001$ \\
\hline Trans-fatty acids & $1 \cdot 8^{\mathrm{a}}$ & 1.6 & $1.9^{a}$ & 1.5 & $1 \cdot 7^{\mathrm{a}}$ & $1 \cdot 1$ & $1.9^{a}$ & $1 \cdot 7$ & 0.676 \\
\hline Cholesterol (mg/d) & $263^{a}$ & 126 & $285^{a}$ & 111 & $279^{a}$ & 130 & $280^{a}$ & 112 & 0.745 \\
\hline Plant sterols $(\mathrm{mg} / \mathrm{d}) \ddagger$ & $256^{a}$ & 116 & $222^{\mathrm{a}}$ & 61 & $247^{a}$ & 69 & $234^{a}$ & 78 & 0.143 \\
\hline Alcohol $(g / d)$ & $4 \cdot 0^{a}$ & $7 \cdot 1$ & $3 \cdot 5^{a}$ & 6.5 & $3 \cdot 2^{a}$ & $6 \cdot 1$ & $3.5^{\mathrm{a}}$ & 6.5 & 0.645 \\
\hline
\end{tabular}

PS, plant sterols.

${ }_{\mathrm{a}, \mathrm{b}}$ Mean values within a row with unlike superscript letters were significantly different from each other $(P<0.05$, Bonferroni's test).

* Data were analysed by using ANOVA for repeated measures. Bonferroni's post hoc comparison test was used for multiple comparisons.

$\dagger$ Energy and fat contents include those provided by the study milk products.

$\ddagger$ Exclusively from background diet. 
MUFA and trans-fatty acids, cholesterol, soluble fibre, alcohol and PS were similar throughout the study period.

Consumption of all milk products was well tolerated and no adverse effects were reported. Compliance was judged as $>95 \%$ in each diet period according to empty milk packages returned to the clinic.

\section{Adiposity measures, blood pressure and blood glucose}

At the end of the run-in period, the study subjects had mean body weight of 72.5 (SD 12.0) kg, BMI 26.6 (SD 3.0$) \mathrm{kg} / \mathrm{m}^{2}$, waist circumference $93.1 \quad(\mathrm{SD} 12.4) \mathrm{cm}$, systolic BP 128 (SD 19) $\mathrm{mmHg}$, diastolic BP 77 (SD 10) $\mathrm{mmHg}$ and fasting blood glucose 900 (SD 12) mg/l. These variables were stable throughout the study. Mean changes during the intervention periods compared to the run-in period ranged from -0.03 to $-0.14 \mathrm{~kg}$ for weight; -0.01 to $-0.05 \mathrm{~kg} / \mathrm{m}^{2}$ for $\mathrm{BMI}$; 0 to $-0.5 \mathrm{~cm}$ for waist circumference; -4.5 to $-4.8 \mathrm{mmHg}$ for systolic BP $(P=0.05) ; 0.4$ to $-2.8 \mathrm{mmHg}$ for diastolic BP; and -9 to $16 \mathrm{mg} / \mathrm{l}$ for blood glucose.

\section{Serum lipid profile}

Serum lipids at the end of the run-in period were: total cholesterol 2640 (sD 310) mg/l, LDL-C 1840 (sD 260) mg/l, HDL-C 530 (sD 130) $\mathrm{mg} / \mathrm{l}$ and TAG 1360 (sD 700) mg/l. Table 3 shows adjusted serum lipid concentrations at the end of each diet sequence and the changes during the two PS milk periods relative to the placebo SM period. After the PS-SM and PSVFM periods, total cholesterol and LDL-C concentrations were significantly $(P<0.015)$ reduced by 7.8 and $6.3 \%$ and by 8.0 and $7.4 \%$, respectively. Changes in HDL-C after the PS-fortified milk (both) were non-significant compared to placebo, but HDL-C rose by $3.8 \%$ after the PS-VFM milk $(P<0.05$ compared to the PS-SM milk). At the end of the PS-VFM period, TAG concentrations decreased by $18.7 \%(P<0.01)$, while there was a non-significant reduction of $13 \cdot 2 \%$ observed with PS-SM. Participants treated with lipid-lowering drugs had lipid responses that were similar to those receiving only dietary treatment (data not shown).

There were moderate correlations between the changes in LDL-C and their concentrations after the placebo period: $r-0.282(P=0.067)$ for the PS-SM period and $r-0.399$ ( $P=0.008$ ) for the PS-VFM period. On the other hand, serum TAG changes were strongly related to concentrations after the placebo milk: $r-0.594(P<0.001)$ for PS-SM and $r-0.518(P<0.001)$ for PS-VFM.

\section{Serum non-cholesterol sterols}

Sitosterol/C after the placebo milk correlated directly with campesterol/C ( $r$ 0.450, $P=0.002)$ and inversely with lathosterol/C $(r-0.374, \quad P=0.014)$. Correlations were weaker when considering absolute non-cholesterol sterol concentrations, with $r$ values of $-0.299(P=0.052)$ for sitosterol $v$. lathosterol and $0 \cdot 298(P=0.053)$ for sitosterol $v$. campesterol. Absolute concentrations of lathosterol and PS as well as their ratios to cholesterol in each treatment period and their changes from the placebo period are shown in Table 4. Both absolute and cholesterol-normalised values of the cholesterol synthesis precursor, lathosterol, increased slightly (range, 5.5-25\%) after the two PS-fortified milk periods, but only the increase of lathosterol/C after the PS-VFM milk was statistically significant $(P<0 \cdot 05)$. On the other hand, campesterol/C and sitosterol/C increased significantly $(P<0.001)$ by $70-75 \%(63-75 \%$ as absolute values) and $106-121 \%$ (91-110\% as absolute values), respectively. Non-cholesterol sterol ratios after the placebo milk correlated with those observed after PS consumption. Thus, for the PS-SM and PSVFM periods, the respective $r$ values were: lathosterol/C, 0.625 and $0.302(P<0.05$, both); campesterol/C, 0.509 and $0.336(P<0.05$, both); and sitosterol/C, 0.529 and 0.649 $(P<0 \cdot 001$, both). The changes of lathosterol/C, campesterol/ $\mathrm{C}$ and sitosterol/C were also correlated between the two PS milk periods, with respective $r$ values of $0.389(P=0.010)$, $0.526(P<0.001)$ and $0.559(P<0.001)$. Similar correlations

Table 3. Serum concentrations of lipids in each dietary period

(Mean values with their standard errors and $95 \%$ confidence intervals)

\begin{tabular}{|c|c|c|c|c|c|c|c|c|c|c|c|}
\hline \multirow[b]{2}{*}{ Serum lipids (mg/l) } & \multicolumn{2}{|c|}{$\begin{array}{l}\text { A: Placebo } \\
\text { skimmed } \\
\text { milk }\end{array}$} & \multicolumn{3}{|c|}{$\begin{array}{l}\text { B: PS- fortified } \\
\text { skimmed milk }\end{array}$} & \multicolumn{3}{|c|}{$\begin{array}{c}\text { C: PS-fortified } \\
\text { semi-skimmed } \\
\text { vegetable-fat milk }\end{array}$} & \multirow{2}{*}{$\begin{array}{c}\text { A-B } \\
\text { PANCOVA }\end{array}$} & \multirow{2}{*}{$\begin{array}{c}\text { A-C } \\
\text { PANCOVA }\end{array}$} & \multirow{2}{*}{$\begin{array}{c}\text { B-C } \\
P \text { ANCOVA }\end{array}$} \\
\hline & Mean & SE & Mean & SE & $95 \% \mathrm{Cl}$ & Mean & SE & $95 \% \mathrm{Cl}$ & & & \\
\hline $\begin{array}{l}\text { Total cholesterol* } \\
\text { Change from placebo } \dagger\end{array}$ & $2560^{b}$ & 37 & $\begin{array}{l}2360^{c} \\
-197\end{array}$ & 37 & $-299,-95$ & $\begin{array}{l}2400^{c} \\
-162\end{array}$ & 37 & $-263,-60$ & 0.0009 & 0.0069 & 0.7782 \\
\hline $\begin{array}{l}\text { LDL-cholesterol }^{*} \\
\text { Change from placebo† }\end{array}$ & $1750^{\mathrm{b}}$ & 31 & $\begin{array}{l}1610^{c} \\
-148\end{array}$ & 31 & $-235,-61$ & $\begin{array}{l}1620^{c} \\
-130\end{array}$ & 31 & $-216,-43$ & 0.0037 & 0.0121 & 0.9082 \\
\hline $\begin{array}{l}\text { HDL-cholesterol } \\
\text { Change from placebo } \dagger\end{array}$ & $5200^{a, b}$ & 70 & $\begin{array}{l}510^{\mathrm{a}} \\
-110\end{array}$ & 70 & $-320,10$ & $\begin{array}{l}540^{\mathrm{b}} \\
-20\end{array}$ & 70 & $-1,40$ & 0.2954 & 0.0624 & 0.0043 \\
\hline $\begin{array}{l}\text { TAG }^{*} \\
\text { Change from placebo } \dagger\end{array}$ & $1440^{a}$ & 69 & $\begin{array}{c}1250^{\mathrm{a}, \mathrm{b}} \\
-190\end{array}$ & 69 & $-381,10$ & $\begin{array}{l}1170^{\mathrm{b}} \\
-267\end{array}$ & 69 & $-458,-77$ & 0.0551 & 0.0075 & 0.4279 \\
\hline
\end{tabular}

PS, plant sterols.

${ }^{a, b}$ Mean values within a row with unlike superscript letters are significantly different from each other by the Tukey-Kramer test $(P<0.05)$.

* Data analysed by using ANCOVA for repeated measures with sex, age, baseline levels, period and treatment sequence and their interactions with treatment as covariates.

The Tukey-Kramer post hoc test was used for multiple comparisons.

† Mean difference ( $95 \%$ confidence interval) from values during the placebo milk period. 
Table 4. Serum cholesterol synthesis and absorption markers, expressed in absolute concentrations $(\mu \mathrm{mol} / \mathrm{l})$ as well as normalised to total cholesterol ( $\mu \mathrm{mol} / \mathrm{mmol})$, in each dietary period (Mean values with their standard errors and $95 \%$ confidence intervals)

\begin{tabular}{|c|c|c|c|c|c|c|c|c|c|c|c|}
\hline & \multicolumn{2}{|c|}{$\begin{array}{l}\text { A: Placebo } \\
\text { skimmed milk }\end{array}$} & \multicolumn{3}{|c|}{ B: PS-fortified skimmed milk } & \multicolumn{3}{|c|}{$\begin{array}{l}\text { C: PS-fortified semi-skimmed } \\
\text { vegetable-fat milk }\end{array}$} & \multirow{2}{*}{$\begin{array}{c}\text { A-B } \\
P \text { ANCOVA }\end{array}$} & \multirow{2}{*}{$\begin{array}{c}\text { A-C } \\
P \text { ANCOVA }\end{array}$} & \multirow{2}{*}{$\begin{array}{c}\text { B-C } \\
P \text { ANCOVA }\end{array}$} \\
\hline & Mean & SE & Mean & SE & $95 \% \mathrm{Cl}$ & Mean & SE & $95 \% \mathrm{Cl}$ & & & \\
\hline \multicolumn{12}{|c|}{ Cholesterol synthesis precursor } \\
\hline $\begin{array}{l}\text { Lathosterol }^{*} \\
\text { Change from placebot }\end{array}$ & $11.99^{a}$ & 0.84 & $\begin{array}{c}12.65^{\mathrm{a}} \\
0.66\end{array}$ & 0.84 & $-1.67,3.00$ & $\begin{array}{c}13.93^{\mathrm{a}} \\
1.94\end{array}$ & 0.84 & $-0.39,4.28$ & 0.8423 & 0.2384 & 0.5321 \\
\hline $\begin{array}{l}\text { Lathosterol/C* } \\
\text { Change from placebo† }\end{array}$ & $2 \cdot 15^{a}$ & $0 \cdot 15$ & $\begin{array}{l}2 \cdot 38^{\mathrm{a}, \mathrm{b}} \\
0 \cdot 23\end{array}$ & 0.15 & $-0.20,0.66$ & $\begin{array}{l}2.68^{\mathrm{b}} \\
0.52\end{array}$ & 0.15 & $0.09,0.95$ & 0.5482 & 0.0496 & 0.3733 \\
\hline Cholesterol absorption mark & & & & & & & & & & & \\
\hline $\begin{array}{l}\text { Campesterol } \\
\text { Change from placebo† }\end{array}$ & $14 \cdot 68^{\mathrm{a}}$ & 1.36 & $\begin{array}{c}23 \cdot 90^{\mathrm{b}} \\
9 \cdot 22\end{array}$ & 1.26 & $6 \cdot 77,11 \cdot 67$ & $\begin{array}{l}25 \cdot 65^{\mathrm{b}} \\
10 \cdot 98\end{array}$ & $1 \cdot 12$ & $8.51,13.44$ & $<0.0001$ & $<0.0001$ & 0.3289 \\
\hline $\begin{array}{l}\text { Campesterol/C* } \\
\text { Change from placebo } \dagger\end{array}$ & $3.06^{a}$ & $0 \cdot 28$ & $\begin{array}{l}5 \cdot 21^{\mathrm{b}} \\
2 \cdot 15\end{array}$ & 0.27 & $1 \cdot 67,2.63$ & $\begin{array}{l}5 \cdot 34^{\mathrm{b}} \\
2 \cdot 28\end{array}$ & 0.23 & $1 \cdot 80,2 \cdot 75$ & $<0.0001$ & $<0.0001$ & 0.8610 \\
\hline $\begin{array}{l}\text { Sitosterol* } \\
\text { Change from placebo† }\end{array}$ & $13 \cdot 25^{a}$ & $1 \cdot 12$ & $\begin{array}{l}25 \cdot 26^{\mathrm{b}} \\
12 \cdot 00\end{array}$ & 0.97 & $9.35,14.65$ & $\begin{array}{l}27 \cdot 77^{\mathrm{b}} \\
14 \cdot 52\end{array}$ & 0.94 & $11 \cdot 76,17 \cdot 27$ & $<0.0001$ & $<0.0001$ & 0.1425 \\
\hline $\begin{array}{l}\text { Sitosterol// } C^{*} \\
\text { Change from placebo } \dagger\end{array}$ & $2 \cdot 54^{a}$ & 0.25 & $\begin{array}{l}5 \cdot 24^{\mathrm{b}} \\
2 \cdot 70^{2}\end{array}$ & 0.21 & $2 \cdot 14,3 \cdot 26$ & $\begin{array}{l}5 \cdot 60^{\mathrm{b}} \\
3.06\end{array}$ & 0.20 & $2.48,3.64$ & $<0.0001$ & $<0.0001$ & 0.4066 \\
\hline
\end{tabular}

PS, plant sterols.

a,b Mean values within a row with unlike superscript letters are significantly different from each other by the Tukey-Kramer test $(P<0.05)$.

* Data analysed by using ANCOVA for repeated measures, with sex, age, baseline levels, period and treatment sequence and their interactions with treatment as covariates. The Tukey-Kramer post hoc test was used for multiple

† Meandifference (95\% confidence interval) from values during the placebo milk period. 
were observed when using absolute instead of cholesterolnormalised non-cholesterol sterol values (data not shown).

In partial correlation models adjusted for LDL-C concentrations after placebo milk and hypolipidaemic drug treatment, LDL-C changes related directly to sitosterol/C after the placebo milk only in the PS-VFM period $(r 0.320, P=0.042)$, but not in the PS-SM period $(r 0.173, P=0.281)$. No correlations were observed for lathosterol/C or campesterol/C with LDL-C changes.

To further assess whether basal serum non-cholesterol sterols as markers of cholesterol synthesis and absorption efficiency were predictive of cholesterol-lowering responsiveness, we plotted (Fig. 1) the mean percentage LDL-C changes for each PS milk period and compared groups above and below the median values of non-cholesterol sterols: $\mathrm{C}$ ratios after the placebo milk. After adjustment for placebo LDL-C levels and hypolipidaemic drug treatment, LDL-C responses to PS-enriched milk consumption were enhanced in participants whose serum lathosterol/C values were below the median, compared to those above the median. Conversely, low basal serum PS:C ratios tended to be associated with blunted LDL-C responses, compared to high ratios.

With the premise that serum non-cholesterol sterol changes after PS consumption would also reflect differences in cholesterol synthesis and absorption, we further examined their predictive power of LDL-C responses. Fig. 2 depicts the individual absolute changes (from corresponding values after the placebo milk period) of serum sitosterol/C and campesterol/C after the PS-VFM period. We divided the groups into subjects with increases of either serum PS ratio above and below the respective medians and calculated the LDL-C responses of each subgroup. As shown, participants disclosing increases of PS ratios above the median were significantly more responsive than those with smaller increases. Similar trends existed after PS-SM intake, with adjusted changes of LDL-C (mean and $95 \% \mathrm{CI}$ ) of $-6 \cdot 7(-12 \cdot 1,-1 \cdot 3) \%$ and -1.9 $(-7 \cdot 4,3.6) \%$, respectively, when the changes of sitosterol/C were above or below the median, and of $-5.9(-11.4$, $-0.4) \%$ and $-2.9(-8.2,2.5) \%$, respectively, when the changes of campesterol/C were above or below the median. When absolute sitosterol and campesterol values were used, similar trends were observed but statistical significance was lost for most associations (data not shown). The changes of lathosterol or lathosterol/C after the intake of the PS-enriched milk (both) had no predictive value of LDL-C responses. That serum PS:C ratios increased in all individual participants (Fig. 2) provides additional proof of compliance with the test milks used in the present study.

\section{Discussion}

The results of this randomised, placebo-controlled, crossover feeding study conducted in subjects with moderate hypercholesterolaemia show that, when compared to consumption of placebo SM, the intake of $2 \mathrm{~g} / \mathrm{d}$ of PS with enriched SM or VFM is associated with similar significant decreases in serum total cholesterol and LDL-C concentrations. Compared to the PS-SM, the increased consumption of PUFA from the vegetable fat-enriched PS-VFM resulted in a similar LDL-C lowering response but an enhanced reduction of serum TAG concentrations. Furthermore, LDL-C responses to PS-enriched milk consumption were variably enhanced in participants with low basal serum lathosterol:C ratios or high basal serum $\mathrm{PS}: \mathrm{C}$ ratios. They were also more pronounced in those with higher post-treatment increases of serum PS:C ratios. As both low-serum lathosterol and high-serum PS are indicative of low cholesterol synthesis and high intestinal cholesterol absorption efficiency ${ }^{(19)}$, these data suggest that subjects with low basal cholesterol synthesis, who are also cholesterol hyper-absorbers, sustain more benefit from an intervention

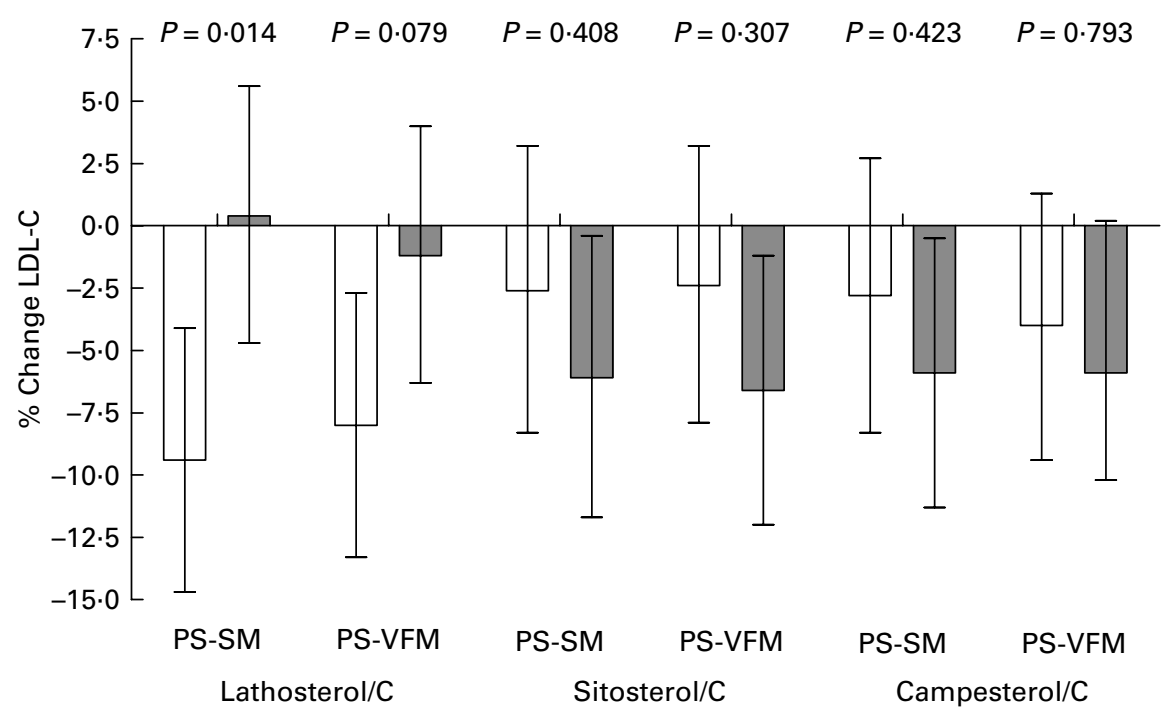

Fig. 1. Mean percentage LDL cholesterol (LDL-C) changes for each plant sterol (PS)-enriched milk intake period $v$. serum lathosterol:cholesterol (C), sitosterol:C and campesterol:C ratios above $(\square)$ and below $(\square)$ the median values after the placebo period $(2 \cdot 05,2 \cdot 27$ and $2.58 \mu \mathrm{mol} / \mathrm{mmol}$, respectively). Values are means with their standard errors represented by vertical bars. Data were analysed by ANCOVA with adjustment for LDL-C concentrations after the placebo period and hypolipidaemic drug treatment. PS-SM, PS-enriched skimmed milk; PS-VFM, PS-enriched semi-skimmed vegetable fat-enriched milk. 

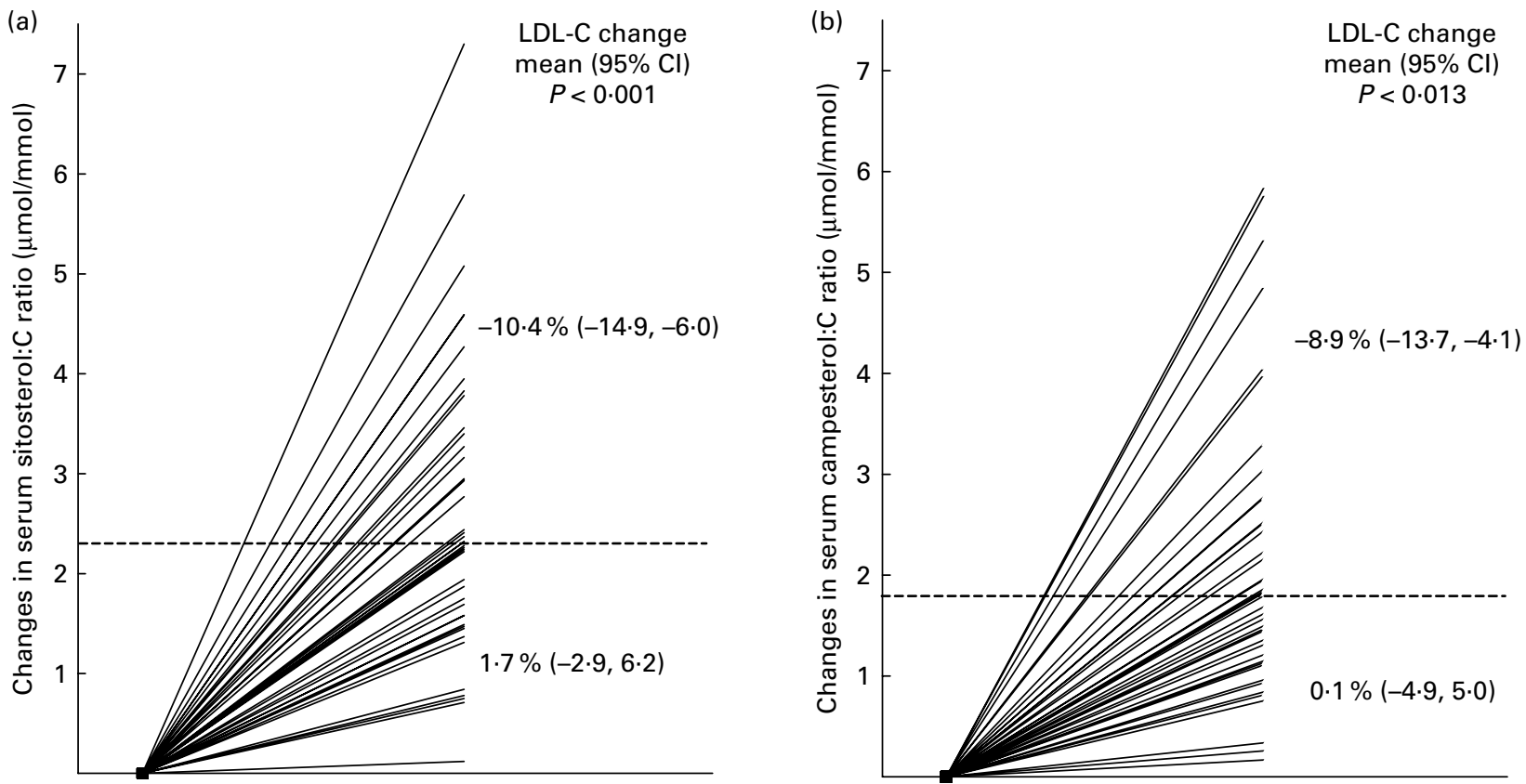

Fig. 2. Mean (plus $95 \% \mathrm{Cl}$ ) percentage LDL-cholesterol (LDL-C) responses in participants $v$. changes in (a) serum sitosterol:cholesterol (C) and (b) campesterol:C ratios above and below the median changes $(2.32$ and $1.81 \mu \mathrm{mol} / \mathrm{mmol}$, respectively), indicated as broken lines. All individual data points start at zero in the $x$-axis because they represent absolute changes from corresponding values after the placebo milk period. Data were analysed by ANCOVA with adjustment for LDL-C concentrations after the placebo period and hypolipidaemic drug treatment.

aimed at inhibiting intestinal cholesterol absorption such as PS intake than those with high basal cholesterol synthesis and reciprocally low intestinal cholesterol absorption efficiency.

In our study, administration of PS in two different low-fat milk formats was associated with an LDL-C-lowering efficacy of approxiamately $8 \%$, close to the average reduction of 9$10 \%$ expected with a $2 \mathrm{~g} / \mathrm{d}$ dose provided in different food formats $^{(3,4)}$ or to LDL-C reductions reported for similar doses delivered with low-fat foods such as milk and beverages ${ }^{(3)}$. One previous study had examined the effects of PS incorporated into VFM at two doses $(1.2$ and $1.6 \mathrm{~g} / \mathrm{d})$, which resulted in LDL-C reductions of 7 and $10 \%$, respectively, in comparison to a control group ${ }^{(20)}$. The higher PUFA content of the PS-VFM added $1.5 \%$ of energy to the overall diet (Table 2). In the absence of other dietary fatty acid or cholesterol changes, such higher dietary PUFA intake would translate into an LDL-C decrease of $13 \mathrm{mg} / \mathrm{l}^{(21)}$, but the LDL-C response to PS was similar with the two PS milk (Table 3). Our study was not powered to detect significant LDL-C changes due to this small increment of dietary PUFA intake. However, consumption of the PS-VFM, but not that of the PS-SM, was associated with a significant $18.7 \%$ lowering of TAG, which, like that of LDL-C, was related to basal lipid levels. No consistent significant changes in TAG have been usually described after PS supplementation, but recent reports suggest that modest reductions occur after plant stanol consumption in relation to baseline levels ${ }^{(13)}$ and in patients with metabolic syndrome associated with elevated TAG ${ }^{(22)}$. As it occurs with statins due to suppressed cholesterol synthesis ${ }^{(23)}$, PS may decrease the mass of hepatic cholesterol available for VLDL production via a lower flux of intestinal cholesterol, with ensuing reduction of circulating $\mathrm{TAG}^{(22)}$.

Although intestinal absorption of PS is low ${ }^{(1)}$, both absolute serum PS concentrations and cholesterol-standardised values, mainly sitosterol and campesterol, do reflect dietary intakes and are thus elevated following a plant-based diet rich in PS $^{(18)}$ or consumption of PS-supplemented foods ${ }^{(23)}$, as was also observed in our study. Serum lathosterol:C ratios increased by $11-25 \%$, which can be explained by small compensatory increases in endogenous cholesterol synthesis ${ }^{(19)}$. Similar large increases in circulating PS concentrations and small increases or no changes in cholesterol synthesis precursors have been observed in numerous short-term clinical studies with PS intake ${ }^{(23,24)}$, as well as in a non-experimental setting after long-term use ${ }^{(25)}$. Whether elevated serum PS concentrations within physiological ranges carry an increased atherogenic risk is a controversial topic ${ }^{(18,23,26-28)}$. If proved to be true, this might weaken the beneficial effect of the cholesterol-lowering effect of PS on cardiovascular risk reduction. Clinical studies with cardiovascular end-points would be necessary to find an ultimate answer to this telling question.

We further examined whether serum concentrations of noncholesterol sterols related to the cholesterol-lowering responsiveness to PS, an important topic for which so far inconsistent results have been reported ${ }^{(9-15)}$. In line with the findings of the elegant studies of Rideout et $a l^{(15)}$, who used kinetic stable-isotope measures of endogenous cholesterol synthesis, we showed that basal cholesterol synthesis, estimated from circulating levels of the cholesterol precursor lathosterol, was inversely associated with the LDL-C response to PS (Fig. 1). Similar findings have been reported by other 
authors $^{(29-31)}$. Conversely, high baseline cholesterol absorption efficiency, as defined by high sitosterol/C and campesterol/C, related directly, albeit non-significantly, to enhanced LDL-C lowering after PS supplementation, which concurs with findings from other studies using PS or plant stanols ${ }^{(9-12)}$, but not with those of other similar studies ${ }^{(13,14)}$. We showed that basal non-cholesterol sterol ratios correlated with those observed after PS consumption. Because higher increases in serum PS following dietary PS intake would potentially be expected in cholesterol hyper-absorbers, we additionally explored the predictive power of post-treatment serum PS changes. Indeed, the LDL-C-lowering response to PS was superior in individuals with higher increases in serum PS, particularly after the PS-VFM period (Fig. 2). Taken together, our findings and those of previous studies ${ }^{(9-12,29-31)}$ suggest that measuring serum non-cholesterol sterols before starting PS treatment might help select individuals with a greater likelihood of optimally responding to PS intervention, namely those characterised by low endogenous cholesterol synthesis and high intestinal cholesterol absorption efficiency. Most probably, variations in genes involved in lipid transport in the enterohepatic circulation are involved in the causal pathway of the inter-individual variability in cholesterol metabolism and lipid responsiveness to PS therapy ${ }^{(7,32-34)}$.

One limitation of this intervention study is that it was conducted under free-living conditions, with individuals following their own rather than a controlled prescribed diet. However, self-reported nutrient intake and applied measures of adherence to the test foods showed good compliance with dietary instructions. Another limitation is the small number of participants enrolled into the study, which was sufficient to demonstrate cholesterol-lowering effects, but blunted the significance of the associations between serum non-cholesterol sterols and LDL-C responses. A further limitation is the inclusion of participants receiving statins, which profoundly affect cholesterol homeostasis $^{(7)}$. However, statin doses were stable throughout the present study, the main results were similar if these participants were excluded, and analyses of non-cholesterol sterols were adjusted for statin use. The study also has the strength of a randomised, double-blind, placebo-controlled, crossover design.

In conclusion, our study confirms that the consumption of PS in the food formats of enriched SM or semi-skimmed VFM in the context of a healthy Mediterranean diet significantly decreased LDL-C in moderate hypercholesterolaemic subjects. We did not observe a greater hypocholesterolaemic effect of combining PS with PUFA-rich vegetable fat in an SM food matrix, but TAG concentrations were lower after consumption of the PS-VFM. Finally, basal serum non-cholesterol sterol concentrations and post-treatment changes in circulating PS were predictive of LDL-C responses to PS consumption.

\section{Acknowledgements}

The authors thank the participants in the present study for their outstanding collaboration. Emili Corbella and Frits Quadt provided excellent statistical assistance. CIBERobn and RTIC 06/0045 are initiatives of ISCIII, Spain. This study was financially supported by grants from Unilever Vlaardingen, the Netherlands; FIS PI06/0365 and RTIC 06/0045 from ISCIII, Spanish Health Ministry; Fundación para la Investigación y Prevención de las Enfermedades Cardiovasculares (FIPEC) and Fundació Privada Catalana de Nutrició i Lípids, Barcelona, Spain. Unilever Vlaardingen also provided the milk products used in the study. The authors' responsibilities were as follows: E. R. designed the study and drafted the manuscript; P. C.-A. drafted the manuscript; M. C. set up the laboratory method of analysis of phytosterols, performed these analyses, and data collection; M. S. and A. P.-H. obtained dietary data from the patients; and E. A. T. and X. P. substantially contributed to the analysis and/or interpretation of the data, and revised the manuscript critically for important intellectual content. E. A. T. is employed by Unilever R\&D, Vlaardingen, the Netherlands, a company that commercialises foods enriched with plant sterols. None of the other authors had a personal or financial conflict of interest.

\section{References}

1. von Bergmann K, Sudhop T \& Lutjohann D (2005) Cholesterol and plant sterol absorption: recent insights. Am J Cardiol 96, 10D-14D.

2. Trautwein EA, Duchateau GSMJE, Lin Y, et al. (2003) Proposed mechanisms of cholesterol-lowering action of plant sterols. Eur J Lipid Sci Technol 105, 171-185.

3. Demonty I, Ras RT, van der Knaap HC, et al. (2009) Continuous dose-response relationship of the LDL-cholesterollowering effect of phytosterol intake. J Nutr 139, 271-284.

4. Talati R, Sobieraj DM, Makanji SS, et al. (2010) The comparative efficacy of plant sterols and stanols on serum lipids: a systematic review and meta-analysis. J Am Diet Assoc 110, $719-726$.

5. Abumweis SS, Barake R \& Jones PJ (2008) Plant sterols/ stanols as cholesterol lowering agents: a meta-analysis of randomized controlled trials. Food Nutr Res 52 (Epublication 18 August 2008).

6. Bosner MS, Lange LG, Stenson WF, et al. (1999) Percent cholesterol absorption in normal women and men quantified with dual stable isotopic tracers and negative ion mass spectrometry. J Lipid Res 40, 302-308.

7. Wang DQ (2007) Regulation of intestinal cholesterol absorption. Annu Rev Physiol 69, 221-248.

8. Miettinen TA, Tilvis RS \& Kesaniemi YA (1990) Serum plant sterols and cholesterol precursors reflect cholesterol absorption and synthesis in volunteers of a randomly selected male population. Am J Epidemiol 131, 20-31.

9. Miettinen TA, Puska P, Gylling H, et al. (1995) Reduction of serum cholesterol with sitostanol-ester margarine in a mildly hypercholesterolemic population. $N$ Engl J Med 333, 1308-1312.

10. Mussner MJ, Parhofer KG, Von Bergmann K, et al. (2002) Effects of phytosterol ester-enriched margarine on plasma lipoproteins in mild to moderate hypercholesterolemia are related to basal cholesterol and fat intake. Metabolism 51, 189-194.

11. Thuluva SC, Igel M, Giesa U, et al. (2005) Ratio of lathosterol to campesterol in serum predicts the cholesterol-lowering effect of sitostanol-supplemented margarine. Int $J$ Clin Pharmacol Ther 43, 305-310.

12. Fuentes F, Lopez-Miranda J, Garcia A, et al. (2008) Basal plasma concentrations of plant sterols can predict LDL-C 
response to sitosterol in patients with familial hypercholesterolemia. Eur J Clin Nutr 62, 495-501.

13. Naumann E, Plat J, Kester AD, et al. (2008) The baseline serum lipoprotein profile is related to plant stanol induced changes in serum lipoprotein cholesterol and triacylglycerol concentrations. J Am Coll Nutr 27, 117-126.

14. Houweling AH, Vanstone CA, Trautwein EA, et al. (2009) Baseline plasma plant sterol concentrations do not predict changes in serum lipids, C-reactive protein (CRP) and plasma plant sterols following intake of a plant sterolenriched food. Eur J Clin Nutr 63, 543-551.

15. Rideout TC, Harding SV, Mackay D, et al. (2010) High basal fractional cholesterol synthesis is associated with nonresponse of plasma LDL cholesterol to plant sterol therapy. Am J Clin Nutr 92, 41-46.

16. Kris-Etherton PM \& Dietschy J (1997) Design criteria for studies examining individual fatty acid effects on cardiovascular disease risk factors: human and animal studies. $\mathrm{Am} \mathrm{J}$ Clin Nutr 65, Suppl. 5, 1590S-1596S.

17. Jiménez-Escrig A, Santos-Hidalgo AB \& Saura-Calixto F (2006) Common sources and estimated intake of plant sterols in the Spanish diet. J Agric Food Chem 54, 3462-3471.

18. Escurriol V, Cofan M, Moreno-Iribas C, et al. (2010) Phytosterol plasma concentrations and coronary heart disease in the prospective Spanish EPIC cohort. J Lipid Res 51, 618-624.

19. Santosa S, Varady KA, AbuMweis S, et al. (2007) Physiologi$\mathrm{cal}$ and therapeutic factors affecting cholesterol metabolism: does a reciprocal relationship between cholesterol absorption and synthesis really exist? Life Sci 80, 505-514.

20. Thomsen AB, Hansen HB, Christiansen C, et al. (2004) Effect of free plant sterols in low-fat milk on serum lipid profile in hypercholesterolemic subjects. Eur J Clin Nutr 58, 860-870.

21. Clarke R, Frost C, Collins R, et al. (1997) Dietary lipids and blood cholesterol: quantitative meta-analysis of metabolic ward studies. BMJ 314, 112-117.

22. Plat J, Brufau G, Dallinga-Thie GM, et al. (2009) A plant stanol yogurt drink alone or combined with a low-dose statin lowers serum triacylglycerol and non-HDL cholesterol in metabolic syndrome patients. J Nutr 139, 1143-1149.

23. Chan YM, Varady KA, Lin Y, et al. (2006) Plasma concentrations of plant sterols: physiology and relationship with coronary heart disease. Nutr Rev 64, 385-402.
24. Naumann E, Plat J \& Mensink RP (2003) Changes in serum concentrations of noncholesterol sterols and lipoproteins in healthy subjects do not depend on the ratio of plant sterols to stanols in the diet. J Nutr 133, 2741-2747.

25. Fransen HP, de Jong N, Wolfs M, et al. (2007) Customary use of plant sterol and plant stanol enriched margarine is associated with changes in serum plant sterol and stanol concentrations in humans. J Nutr 137, 1301-1306.

26. Patel MD \& Thompson PD (2006) Phytosterols and vascular disease. Atherosclerosis 186, 12-19.

27. Weingartner O, Bohm M \& Laufs U (2009) Controversial role of plant sterol esters in the management of hypercholesterolaemia. Eur Heart J 30, 404-409.

28. Silbernagel G, Fauler G, Hoffmann MM, et al. (2010) The associations of cholesterol metabolism and plasma plant sterols with all-cause and cardiovascular mortality. J Lipid Res 51, 2384-2393.

29. Gylling H, Radhakrishnan R \& Miettinen TA (1997) Reduction of serum cholesterol in postmenopausal women with previous myocardial infarction and cholesterol malabsorption induced by dietary sitostanol ester margarine: women and dietary sitostanol. Circulation 96, 4226-4231.

30. Gylling H, Puska P, Vartiainen E, et al. (1999) Serum sterols during stanol ester feeding in a mildly hypercholesterolemic population. J Lipid Res 40, 593-600.

31. Carr TP, Krogstrand KL, Schlegel VL, et al. (2009) Stearateenriched plant sterol esters lower serum LDL cholesterol concentration in normo- and hypercholesterolemic adults. J Nutr 139, 1445-1450.

32. Plat J, Bragt MC \& Mensink RP (2005) Common sequence variations in ABCG8 are related to plant sterol metabolism in healthy volunteers. J Lipid Res 46, 68-75.

33. Zhao HL, Houweling AH, Vanstone CA, et al. (2008) Genetic variation in $\mathrm{ABC}$ G5/G8 and NPC1L1 impact cholesterol response to plant sterols in hypercholesterolemic men. Lipids 43, 1155-1164.

34. De Castro-Oros I, Pampin S, Cofan M, et al. (2011) Promoter variant $-204 \mathrm{~A}>\mathrm{C}$ of the cholesterol 7alpha-hydroxylase gene: Association with response to plant sterols in humans and increased transcriptional activity in transfected HepG2 cells. Clin Nutr 30, 239-246. 